\title{
BUSINESS
}

\section{Smart networking}

\section{Theoreticians have combined their expertise to form a network to help other scientists design materials or understand biological pathways. Katharine Sanderson reports.}

F

or the past three months Joerg Schaefer, a physicist at the University of Würzburg in Germany, has been waiting for a group of theoretical spectroscopists to calculate the exact position of gold and platinum atoms in nanowires made from single atoms of the metals. When laid out on a semiconductor surface, the wires could potentially be used to transfer data at high speeds in microprocessors by acting as switches. Once the theoreticians have worked out what combination of metal and semiconductor should produce a wire that can be changed at will from insulator to conductor, Schaefer will try to make that combination in the lab, atom by atom.

Theoretical spectroscopy simulates how electrons in molecules behave and can predict what the molecule's spectra will look like. Schaefer can't do the calculations himself, he says, because he doesn't have the necessary tools or knowledge. But he hopes that the theoretical spectroscopists will back up what he is seeing in his lab. "If everything goes right," he says, "the real world and their elusive phantom world will come to the same result."

Schaefer is one of the first clients of the European Theoretical Spectroscopy Facility (ETSF), a network created earlier this year. The network can investigate anything from how the structure of a protein affects its properties to the likely properties of a new material.

Established mainly as a service for academic collaboration, its sponsors hope that it will evolve into a service for sale to industry.

\section{Designer tools}

The network simulates observational tools such as nuclear magnetic resonance, which shows how certain nuclei react to a magnetic field; infrared or Raman spectroscopy, which look at the vibration of molecules; and photoemission spectroscopy, which measures the distribution of electrons in substances. Its supporters say that it will help to save time by predicting what properties new materials or molecules will have before experimentalists try to make them - a time-consuming process.

The facility grew out of Nanoquanta, a similar network funded by the European Commission's Sixth Framework Programme, but

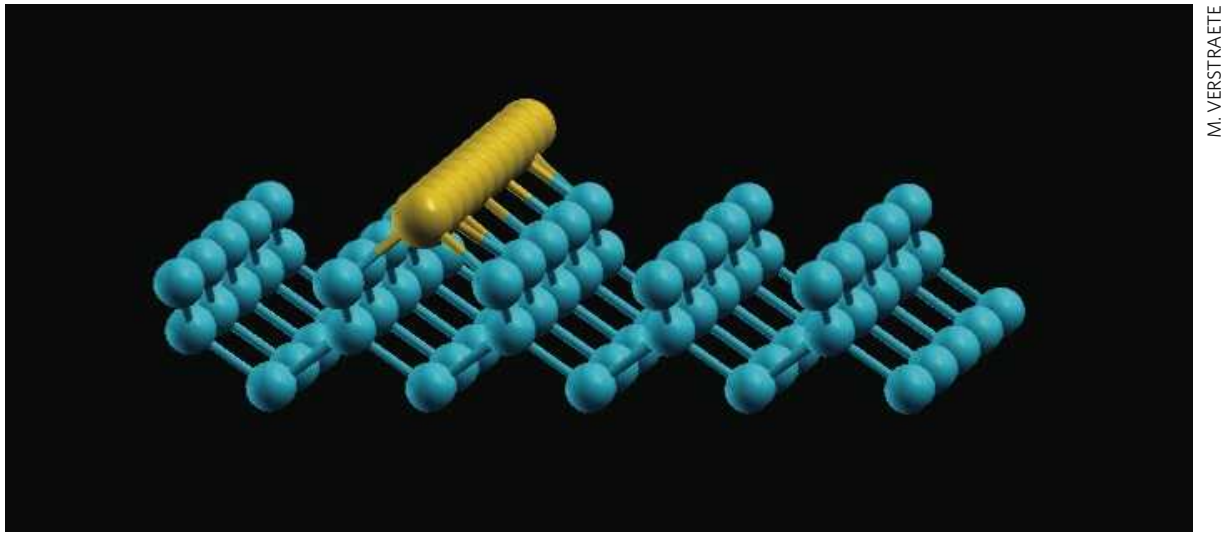

Spectroscopists want to know if gold nanowires (yellow) on silicon surfaces will speed up data transfer.

focused more on building its own research collaborations than on offering a service to others. Nanoquanta will run to the end of 2008 and is coordinated by Rex Godby from the University of York, UK. Both networks tackle "the theory and simulation of all sorts of characterization techniques that involve electrons", says Godby.

The ETSF doesn't have a physical home, but it involves about 120 scientists spread across 10 research institutions that are hubs for different spectroscopic techniques. To tap into the network, potential users submit a proposal that outlines their problem.

The first call for proposals, earlier this year, attracted 54 submissions from researchers in academia and industry, of which the ETSF says it will back 21 .

Industrial proposals made up $15 \%$ of the submissions. One of these was from Antonio Porro, head of the Centre for Nanomaterials Applications in Construction, a firm run by Labein, a contract research and development company in Bilbao, Spain. Porro is looking at the fine structure of cements, and uses simulations to speed up the process of designing lighter, stronger materials. "We can cut by half the process of developing a material experimentally," he says.

The industrial bent of the network is mirrored by a shift in its funding as it evolves from Nanoquanta to the ETSF. Nanoquanta's funding comes from the European Commission's research directorate, which is more interested in blue-skies research. The ETSF's main initial funding, however, is being provided by the more industry-linked information society and media directorate, to the tune of $€ 3.7$ million (US\$5.4 million).

\section{Service update}

For now, the network remains very much an academic pursuit. "The aim is not to make money, it is to provide a service," says Lucia Reining, a physicist at the École Polytechnique in Paris who is the ETSF's chairwoman. But that could change. From now on the ETSF will ask for proposals every six months, and during the next couple of rounds it wants to gauge the interest from industry. "We are anxious to discover how much companies are willing to pay," says Godby.

Godby says that the ETSF has no immediate plans to spawn a start-up company: "It would be wrong for the evolution of the ETSF to be driven by our commercial users," says Godby. But a spokesman for the information society and media directorate-general says that any of the ETSF's projects that went on to produce a spin-off company would be regarded as a great success.

And Porro sees a genuine business opportunity for the venture. The ETSF "can have a specific commercial aspect", he says. "Normally, the materials design process is fully experimental," Porro says. Being able to predict and validate properties early on is a key aspect of the process, he says, and rigorous theoretical models should help with that. "This way we can save a huge amount of time and money." 